\title{
The Relative Viscosity of Concentrated Rubber Suspensions and Viscoelastic Modulus of 3D Cross-Linked Elastomers Filled with Solid Particles
}

\author{
Alexandr Ermilov, Ergasch Nurullaev \\ Perm National Research Polytechnic University, Perm, Russia \\ Email: ergnur@mail.ru
}

Received July 29, 2013; revised September 1, 2013; accepted September 8, 2013

Copyright (C) 2013 Alexandr Ermilov, Ergasch Nurullaev. This is an open access article distributed under the Creative Commons Attribution License, which permits unrestricted use, distribution, and reproduction in any medium, provided the original work is properly cited. In accordance of the Creative Commons Attribution License all Copyrights (C) 2013 are reserved for SCIRP and the owner of the intellectual property Alexandr Ermilov, Ergasch Nurullaev. All Copyright (C) 2013 are guarded by law and by SCIRP as a guardian.

\begin{abstract}
For the first time, this paper describes the concentration dependence of the relative dynamic viscosity coefficient of rubber suspensions and the initial viscoelastic modulus of 3D cross-linked elastomers on the maximum volume filling with solid polydisperse particles. It allows to predict the rheological and mechanical properties of the polymer compositions being developed now. In this paper, we present the first experimental study of the pole of the concurrent lines of the concentration dependence in the coordinates of the linear form. The pole validates the invariant value of the constant of the developed equation and allows the experimental determination of the maximum volume filling of polymer binders filled with separate fractions or polydisperse mixtures. The results of the study are recommended for use in developing new polymer composite materials.
\end{abstract}

Keywords: Viscosity; Mechanical Destruction; Elastomeric Composites with Dispersed Fillers; Rheology; Rubbers; Polymeric Binders; Asphalt Coatings

\section{Introduction}

Engineering prediction of the coefficient of dynamic viscosity $(\eta)$ and the initial viscoelastic modulus $(E)$ of low-molecular-weight rubbers and 3D cross-linked elastomers based on themin relation to the maximum (or limiting) volume filling $\left(\varphi / \varphi_{m}\right)$ is of great importance in the development and manufacture of new polymer composite materials.

The degree of influence of the volume fraction of solid particles in the filler $(\varphi)$ is known to be related to the maximum filler volume fraction determined by the shape and fractional composition of the particles, as well as their physicochemical interaction with the molecules of the polymer binder, optionally containing a plasticizer [1].

Rutgers analyzed about 100 empirical formulas of the concentration dependence of the coefficient of dynamic viscosity of various suspensions [2].

Let us note the most frequently used equations that adequately describe the dependencies for suspensions:

Eilers [3] proposed a formula for the highly-concen- trated suspensions on the basis of experimental data:

$$
\eta_{r}=\frac{\eta_{f}}{\eta_{o}}=\left(1+\frac{k \varphi}{1-s \varphi}\right)^{2}
$$

where the parameters $k$ and $s$ depend on the composition of the compound and the physical and chemical nature of the components, the superscripts $o$ and $f$ refer to unfilled and filled elastomeric binders, respectively. The value of $k$ varied in the range of $0.75-1.25$, the value of $s$ for the spherical particles ranged from 1.20 to 1.35 . Further studies confirmed the relation $s=1 / \varphi_{m}$, where $\varphi_{m}$ is the maximum volume filling.

Mooney [4] used his own formula:

$$
\eta_{r}=\exp \left(\frac{k \varphi}{1-s \varphi}\right),
$$

in which the same parameters ( $k$ and $s$ ) are determined by the physical and chemical features of the filled suspensions.

Chong et al. [5] and Fedors [6] summarized the ex- 
periments with various polymer suspensions and 3D cross-linked filled elastomers and got an empirical formula for the coefficient of dynamic viscosity and the initial viscoelastic modulus:

$$
\eta_{r}=\frac{\eta_{f}}{\eta_{o}}=E_{r}=\frac{E_{f}}{E_{o}}=\left(1+1.25 \frac{\varphi / \varphi_{m}}{1-\varphi / \varphi_{m}}\right)^{2},
$$

where the relations $k=1.25 / \varphi_{m}$ and $s=1 / \varphi_{m}$ are observed.

The aim of this paper is to provide a theoretical justification of the empirical Equation (1) for the dependence of the relative viscosity of concentrated rubber suspendsions and the viscoelastic modulus of 3D cross-linked elastomers filled with solid particles. We also aim to check whether the equation agrees with the experimental data and apply it for the determination of the maximum volume filling of the polymer binder with separate fractions and polydisperse mixtures of solid particles.

\section{Theoretical Study}

We will consider solid particles of arbitrary shapes and fractional composition distributed randomly but uniformly in the matrix, for example, low-molecular-weight rubber, optionally containing a plasticizer. In the mixing process, at a higher volume concentration smaller particles are pushed into the gaps between the larger particles. This increases the effect of the packing density of the particles of the initial poly fractional dispersed filler on the average size layer of the polymer binder between the particles at a constant value of $\varphi$.

Let us denote the coefficient of increasing dynamic viscosity of the initial rubber or the initial viscoelastic modulus of the 3D cross-linked elastomer as

$$
R_{f}=\eta_{f} / \eta_{o}=E_{f} / E_{o} .
$$

In the first approximation, the value of $R_{f}$ is proportional to the ratio of the filler volume fraction to the volume fraction of the polymer binder- $\varphi /(1-\varphi)$. However, due to the finite particle size the coefficient of strengthening tends to infinity at $\varphi=\varphi_{m}$, rather than at $\varphi=1$. Therefore, the argument for the function $R_{f}$ should be the ratio $\left(\varphi / \varphi_{m}\right) /\left(1-\varphi / \varphi_{m}\right)=z$. Let the parameter $z$ be a new variable of the given dependence, which allows to generate a differential equation describing the concentration rate of variation of the coefficient:

$$
\frac{d R_{f}}{d z}=C_{1}+C_{2} z
$$

where $C_{1}$ and $C_{2}$ are the unknown coefficients of the linear part of the equation in a general form.

While $\varphi / \varphi_{m}$ varies from 0 to 1 , the value of $z$ as a linear fractional function varies from 0 to $\infty$, which corresponds to the experimental data. In its turn, the indefinite integration of the Equation (2):

$$
R_{f}=\int\left(C_{1}+C_{2}\right) \mathrm{d} z
$$

leads us to the corresponding algebraic expression:

$$
R_{f}=C_{o}+C_{1} z+0.5 C_{2} z^{2}
$$

Taking into account the boundary conditions

$$
\left(C_{o}=R_{f}=1 \text { when } z=0\right)
$$

and the preliminary conditions

$$
C_{1}=2 K \text { and } C_{2}=2 K^{2}
$$

( $K$ is a combined parameter), we can use the expression (3) to obtain a quadratic equation as a polynomial of the second degree:

$$
R_{f}=1+2 K z+K^{2} z^{2}=(1+K z)^{2},
$$

which formally corresponds to the empirical formulas proposed by Chong et al. (1971)-(5) and Fedors (1979)(6):

$$
R_{f}=\left(1+K \frac{\varphi / \varphi_{m}}{1-\varphi / \varphi_{m}}\right)^{2}
$$

The value of Kas a combined parameter was determined on the basis of the rheological experimental data. To do this, the Formula (5) was used in the linear form:

$$
\frac{1}{\sqrt{R_{f}}-1}=-\frac{1}{K}+\frac{\varphi_{m}}{K} \cdot \frac{1}{\varphi}
$$

The results of the experiments obtained by using the expression (6) are shown in Figure 1 as

$$
\left(\sqrt{R_{f}}-1\right)^{-1}=f(\varphi)^{-1}
$$

with respect to the relative coefficient of dynamic viscosity $\left(\eta_{r}=R_{f}\right)$ for the compositions based on low-mo lecular-weight polybutadiene SKD-KTR grade rubber with terminal carboxyl groups filled with silica $\left(\mathrm{SiO}_{2}\right)$ of varying degree of fineness: $1-1 \mu \mathrm{m} ; 2-5 \mu \mathrm{m} ; 3-15$ $\mu \mathrm{m} ; 4-240 \mu \mathrm{m} ; 5-600 \mu \mathrm{m} ; 6$ - a mixture of 2 or 3 fractions taken in the optimal ratio in terms of the minimum porosity for increasing the maximum volume filling of the composition $\left(\varphi_{m}\right)$.

Correlation analysis shows that the most probable value of the combined parameter $K($ at $\mathrm{P}=0.95)$ at the pole of the concurrent lines in the given coordinates equals to 1.25 , whereas $C_{1}=2 K=2.5$. The presence of the "pole" is indicative of the invariant nature of the concentration dependence. It is interesting to note that the Formulas (1) and (5) can be transformed into the wellknown theoretical Einstein's formula for viscosity of dilute suspensions [7]:

$$
\eta_{f}=\eta_{o}(1+2.5 \varphi) \text { at } \varphi_{m}=1 \text {. }
$$

A theoretical justification of the dependence of the relative viscosity of concentrated rubber suspensions and 


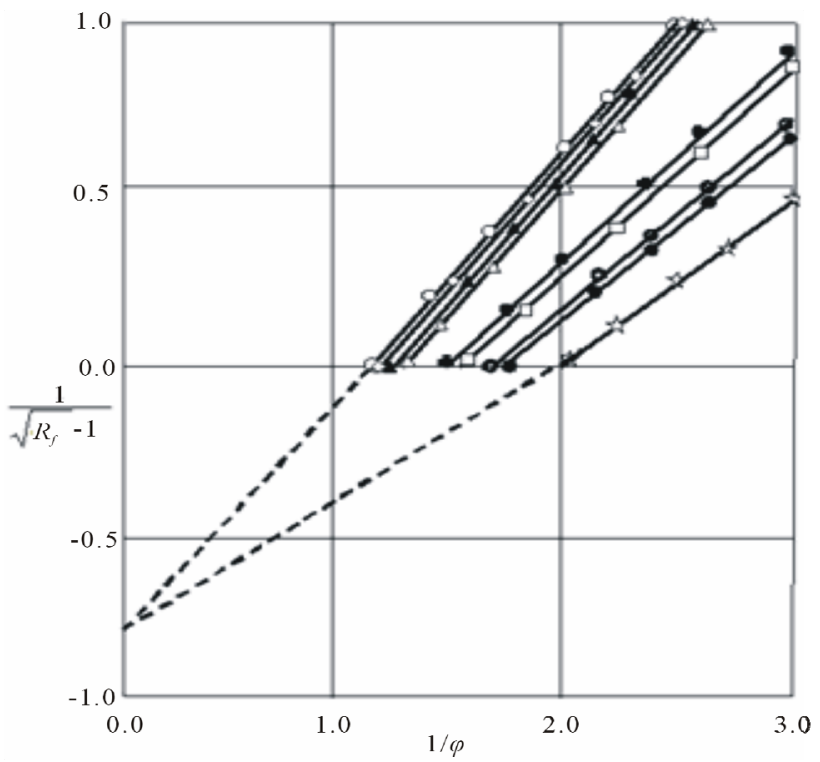

Figure 1. The dependence $\left(\sqrt{R_{f}}-1\right)^{-1}=f(\varphi)^{-1}$ for the compositions based on low-molecular-weight polybutadiene SKD-KTR grade rubber with terminal carboxyl groups filled with silica $\left(\mathrm{SiO}_{2}\right)$ of varying degree of fineness: $\mu \mathrm{m}$; $-5 \mu \mathrm{m}$; $2-15 \mu \mathrm{m} ; \square-240 \mu \mathrm{m} ;-600 \mu \mathrm{m}$; $\triangle \wedge \quad O_{-}$a mixture of 2 or 3 fractions taken in the optimal ratio in terms of the minimum porosity for increasing the maximum volume filling of the composition $\left(\varphi_{m}\right)$.

the viscoelastic modulus of 3D cross-linked elastomers filled with solid particles has been provided for the first time. We have also detected the pole which validates the invariant value of the constant of the developed equation.

Thus, the theoretical dependence (5) corresponds to the Formula (1), which (alongside with our experimental data and the results of Chong et al. [5] and Fedors [6] is recommended for use in calculating the coefficient of reinforced rubber suspensions and the viscoelastic modulus of 3D cross-linked elastomers filled with solid particles.

\section{Experimental Study}

Ermilov et al. [8] verified the Formula (1) in terms of enhancement of the initial viscoelastic modulus of 3D cross-linked elastomers for developing a frost-resistant waterproofing rolled material for asphalt coatings.

Various low-molecular-weight rubbers (polyethylene butyl formal, polyesterurethane, polybutadiene, polydieneepoxy urethane) with terminal functional groups

$$
\left(-\mathrm{SH},-\mathrm{OH},-\mathrm{COOH},-\mathrm{CH}(\mathrm{O}) \mathrm{CH}_{2}\right)
$$

and high-molecular-weight unsaturated rubbers (polyisoprene butyl, polyisoprenedivinyl) three-dimensionally crossed-linked by means of the functional groups, in- cluding double bonds $(-\mathrm{CH}=\mathrm{CH}-)$, were used as polymer binders.

The cross-linking agents were compounds having the antipodal functional groups capable of forming the necessary chemical bonds. For example, the reaction between the isocyanate group and the hydroxyl one results in the urethane group which links two molecules of the polymer. Some high-molecular-weight and low-molecular-weight rubbers contained a plasticizer as a component of the polymer binder.

It should be noted that the formation of the elastic and viscous components of the initial modulus at a uniaxial stretching (elongation) of the specimens was as follows. The concentration of the transverse chemical bonds in the $3 \mathrm{D}$ cross-linked polymer binder determined the elastic component of the modulus, whereas the degree of polarity of molecular groups of the binder components (including plasticizer) determined the viscous component of the modulus. In contrast to the elastic component, the viscous component of the modulusis in inverse proportion to temperature and strain rate due to the nature of intermolecular interactions [1].

The dispersed filler was a three-fraction mixture of silica (river quartz sand) with the optimal size grading $(600: 240: 15) \mu \mathrm{m}=(50: 30: 20) \%$. The fraction of $15 \mu \mathrm{m}$ (as well as the other fractions $1 \mu \mathrm{m}$ and $5 \mu \mathrm{m}$ ) was obtained by grinding quartz sand in the vacuum grinder.

Figure 2 shows the calculated dependence:

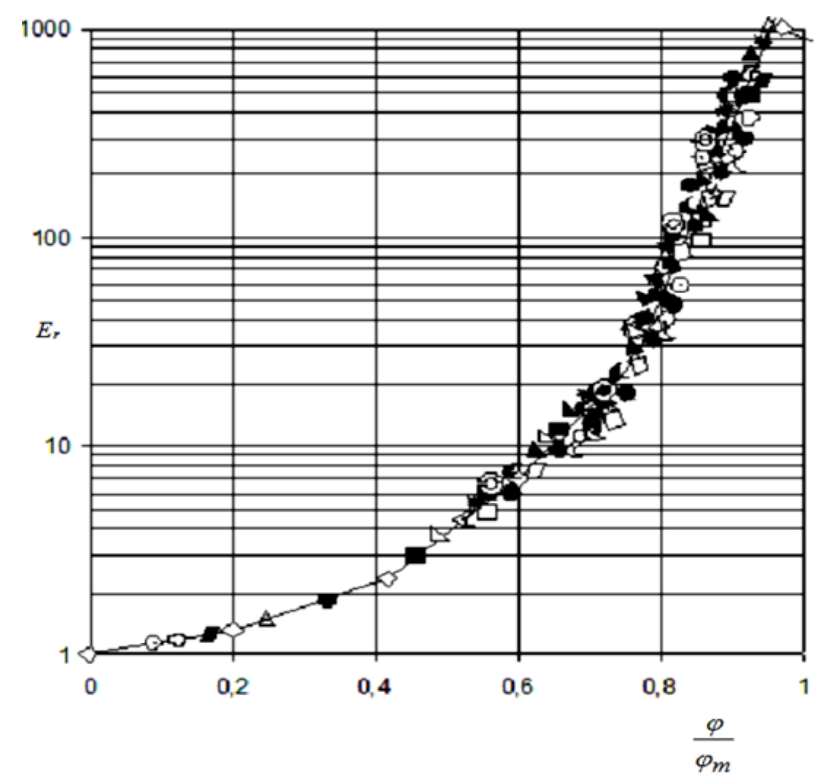

Figure 2. shows the calculated dependence $E_{r}=E_{f} / E_{o}=f\left(\varphi / \varphi_{\mu}\right)$ and the experimental data for filled elastomers based on these rubbers:

1) $(\sqsupset, \square, ڤ \Delta)$ polyethylene butyl formal 2$)(\square, \square, \square)$ polyester urethane, 3) (综) polybutadiene, 4) $(\Delta, \Delta, \Delta)$ polyisoprene butyl, 5) $(\odot, \bullet)$ polyisoprenedivinyl, 6) $(\varangle<\odot)$ polydiene epoxy urethane filled with polydisperse silica. 


$$
E_{r}=E_{f} / E_{o}=f\left(\varphi / \varphi_{m}\right)
$$

and the experimental data for filled elastomers based on these rubbers. It is evident that the result of the experimental verification of the theoretical approach is in agreement with the data of Fedors [6] and Chong et al. [5] both in terms of the dynamic viscosity coefficient (Figure 1) and the relative initial viscoelastic modulus of different polymer compositions (Figure 2).

\section{Engineering Applications}

The formula of reinforcement of rubbers and 3D crosslinked elastomers (1) allows, alongside with the calculation methods proposed by Ermilov and Fedoseev [9], to determine the value of the maximum filling of the polymer binder with separate filler fractions or polydisperse fractions based on them and to minimize the number of the experiments. Indeed, the transformation of the Equation (1) in relation to $\varphi_{m}$ results in the following formula:

$$
\varphi_{m}=\frac{1.25 \varphi+\left(\sqrt{\eta_{f} / \eta_{o}}-1\right) \varphi}{\sqrt{\eta_{f}+\eta_{o}}},
$$

which allows to estimate the value of $\varphi_{m}$ by aviscometric method. This provides a more accurate estimation of the maximum filling compared to determination of the packing density of the particles in bulk since it "automatically" takes into account the intermolecular interaction at the filler-binder interface. Therefore, the most appropriate binder is low-molecular-weight polybutadiene SKD-KTR grade rubber with terminal carboxyl groups of medium polarity in terms of the immobilizing effect of the dispersed filler on the molecular mobility of the binder. The coefficients of dynamic viscosity of the binders and filled compositions based on them were determined using Heppler consistometer at one of the moderate values of $\varphi$ and $3-5$ parallel measurements. The levels of the gradients of shear rate and experimental temperatures were selected in compliance with practical needs and interests of the particular branches of science and industry.

Table 1 presents the results of viscosimetric determination of the maximum filling of polybutadiene rubber with various fractions of silica used in the experiments.

It is clear that with increase in the degree of mechanical grinding (fractions $1,5,15 \mu \mathrm{m}$ ) the value of $\varphi_{m}$ decreases significantly, apparently due to the more angular particles. The fractions $240 \mu \mathrm{m}$ and $600 \mu \mathrm{m}$ screened out from river quartz sand feature particles of more rounded shapes, which are more favorable in shear flows of the compositions.

Nielsen [1] studied the physical properties and the chemical structure of the polymeric base of the binder and plasticizer and concluded that they affect the intermolecular interaction at the filler-binder interface. As a result of the immobilizing effect, the mobility of the molecules at the filler-binder interface decreases, which reduces the value of the maximum volume filling, allother conditions being equal. Therefore, less polar molecules of the binder components show higher values of $\varphi_{m}$.

Table 2 presents the immobilizing effect of the physical and chemical properties of the polymeric binder on its maximum filling with a three-fraction mixture of silica with the optimal size grading $(600: 240: 15) \mu \mathrm{m}=(50: 30$ : $20) \%$.

\section{Conclusions}

1) For the first time, we have presented a theoretical justification and an experimental validation of the formula (1) which invariantly describes the concentration dependence of reinforcement of rubbers and 3D crosslinked elastomers. We have also first located the "pole" of the experimental data of the Equation (5) in the coordinates of the linear form(6) that substantiates the value of the constant $(K=1.25)$.

2) On the basis of the obtained dependence we have developed a viscometric method of determining the maximum volume filling of polymer binders (7) with solid particles of varying fractional composition.

Table 1. The maximum filling of low-molecular-weight polybutadiene SKD-KTR grade rubber with various fractions of silica.

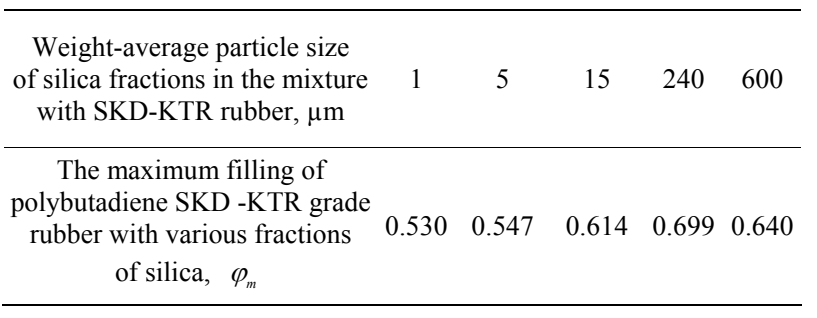

Table 2. The influence of physicochemical features of the polymer binder on its maximum filling with polydispersesilica.

\begin{tabular}{cc}
\hline $\begin{array}{c}\text { Polymer binder filled with a three-fraction } \\
\text { mixture of silica }\end{array}$ & $\begin{array}{c}\text { Maximum volume } \\
\text { filling, } \varphi_{m}\end{array}$ \\
\hline P-9A grade polyester & 0.770 \\
NVTS-2 grade polyethylene butyl & 0.810 \\
$\quad$ formal & 0.830 \\
$\begin{array}{c}\text { Polydieneepoxy urethane PDI-3B grade rubber } \\
\text { plasticized by dioctylsebacate (30\%) }\end{array}$ & 0.910 \\
$\begin{array}{c}\text { BK grade polyisoprene butyl plasticized by } \\
\text { transformer oil (80\%) }\end{array}$ & 0.940 \\
$\begin{array}{c}\text { SKID-L grade polyisoprenedivinyl plasticized } \\
\text { by transformer oil (70\%) }\end{array}$ \\
\hline
\end{tabular}


The use of computational methods for the determination of the maximum filling of the polymer binder with polydisperse mixtures as the initial viscometric values of the maximum filling with separate fractions significantly increased accuracy.

3) The experiments showed that less polar molecules of the binder components provide the value of the maximum filling of the elastomeric binder with three-fractional mixtures of silica at the level of 0.95 , all other conditions being equal. This reduces the coefficient of dynamic viscosity of the filled elastomer and its initial viscoelastic modulus in a 3D cross-linked state.

4) The results of the studies are recommended for use in the accelerated development and optimization of new formulations of polymeric composite materials using mathematical methods, for example, the simplex-lattice design for experiments.

\section{REFERENCES}

[1] L. E. Nielsen, "Mechanical Properties of Polymer and Polymer Composition," Chemistry, 1978, p. 310.

[2] J. R. Rutgers, "Relative Viscosity and Concentration," Rheologica Acta, Vol. 2, No. 4, 1962, pp. 305-348. http://dx.doi.org/10.1007/BF01976051

[3] H. Eilers, "Die Zahigkeiteinekonzentrieren Suspensionen," Kolloid-Zeitschrift, Vol. 97, No. 3, 1941, pp. 313-317.
http://dx.doi.org/10.1007/BF01503023

[4] M. Mooney, "Rheology of Concentration Suspensions," Journal of Colloid Science, Vol. 6, No. 2, 1951, pp. 162156. http://dx.doi.org/10.1016/0095-8522(51)90036-0

[5] J. S. Chong, E. B. Christiansen and A. D. Baer, "Rheological Properties of Concentration Suspensions," Journal of Applied Polymer Science, Vol. 15, No. 8, 1971, pp. 2007-2024.

http://dx.doi.org/10.1002/app.1971.070150818

[6] R. Fedors, "Effect of Filler on the Mechanical Behaviour of Elastomers. Relationships between the Small Strain Modulus and the Type and Concentration of Filler," Polymer, Vol. 20, No. 3, 1979, pp. 324-328. http://dx.doi.org/10.1016/0032-3861(79)90095-8

[7] A. Einstein, "Viscosity of the Dilution Suspensions," Kolloid-Zeitschrift, Vol. 27, No. 3, 1920, pp. 137-139. http://dx.doi.org/10.1007/BF01437675

[8] A. S. Ermilov, E. M. Nurullaev and V. N. Alikin, "Waterproof Frost-Resistant Road Asphalt Coating," Patent Number 2473581, Russian Federation, 2011.

[9] A. S. Ermilov and A. M. Fedoseev, "CombinatorialMultiplicative Method of Calculating the Limiting Filling of Composites with Solid Dispersed Components," Journal of Applied Chemistry, Vol. 77, No. 7, 2004, pp. 12181220 . 